\title{
Neighbourhood walkability and its particular importance for adults with a preference for passive transport
}

\author{
Delfien Van Dyck $^{\mathrm{b}, *}$, Benedicte Deforche ${ }^{\mathrm{b}}$, Greet Cardon $^{\mathrm{a}}$, Ilse De Bourdeaudhuij ${ }^{\mathrm{a}}$ \\ ${ }^{\text {a }}$ Faculty of Medicine and Health Sciences, Department of Movement and Sports Sciences, Ghent University, Watersportlaan 2, B-9000 Ghent, Belgium \\ ${ }^{\mathrm{b}}$ Fund for Scientific Research Flanders (FWO), Belgium
}

\section{A R T I C L E I N F O}

\section{Article history:}

Received 8 April 2008

Received in revised form

27 August 2008

Accepted 29 August 2008

\section{Keywords:}

Neighbourhood walkability

Physical activity

Active transport

Intention

Preference

\begin{abstract}
A B S T R A C T
In this study, differences in physical activity between adults living in high versus low walkable neighbourhoods were examined. In Sint-Niklaas, Belgium, neighbourhood walkability was defined by geographical map data and observations. One high walkable and one low walkable neighbourhood were selected. A sample of 120 adults between 20 and 65 years old, agreed to participate in the study and wore a pedometer for seven days. Self-reported physical activity and psychosocial data were collected. Results showed that residents of the high walkable neighbourhood took more steps/day and walked more for transport in their neighbourhood. Further analyses showed that living in a high walkable neighbourhood was associated with taking more steps, especially in adults with a preference for passive transport and/or a low intention to walk or cycle. In a health promotion context, these results are very promising.
\end{abstract}

(c) 2008 Elsevier Ltd. All rights reserved.

\section{Introduction}

Despite the considerable health benefits of physical activity of moderate intensity, the prevalence of sedentary living habits among adolescents, adults and older people in Europe, as well as in other parts of the world, is relatively high. Depending on the country, approximately $30 \%-60 \%$ of the population does not engage in sufficient levels of physical activity to achieve health benefits (US Department of Health and Human Services [USDHHS], 1996; European Commission, 2003; Haskell et al., 2007). Consequently, the promotion of physical activity participation is a public health priority. However, in order to develop effective interventions, the correlates of physical activity need to be identified (Dishman and Sallis, 1994). Demographic and psychosocial correlates have been studied extensively in adults, but the explanatory power of models including only demographic and psychosocial factors, remains limited (Sallis and Owen, 1999). Also, interventions focusing only on psychosocial determinants have limited long term success (King et al., 1995).

Ecological models posit that physical activity behaviour depends on psychological, sociocultural, policy and physical environmental factors, and that a multilevel approach is needed for behaviour change (Sallis and Owen, 2002). Recently, several researchers have emphasised the importance of physical environmental determinants of physical activity and an increasing number of studies have

\footnotetext{
* Corresponding author. Tel.: +32926463 23; fax: +3292646484.

E-mail address: delfien.vandyck@ugent.be (D. Van Dyck).
}

focused on these factors (Owen et al., 2007; Wendel-Vos et al., 2007). However, thus far, environmental correlates remain the least understood of the physical activity determinants which have been recognised in the literature (Sallis and Owen, 1999).

Among physical environmental determinants, neighbourhood 'walkability' is often evaluated in the literature. Key elements of a 'high walkable' neighbourhood are high street connectivity, high land use mix and high residential density (Frank et al., 2005; Leslie et al., 2007). These elements can be assessed objectively using geographic information systems (GIS) software or neighbourhood observational data (Forsyth et al., 2007; Leslie et al., 2007). Previously, walkability and its link with active transport as a component of physical activity, was only studied in transportation and urban planning literature. Recently, several studies in the public health literature have also investigated the relationship between neighbourhood walkability and physical activity in adults (Frank et al., 2005; Owen et al., 2007; Frank et al., 2006; Saelens et al., 2003a; Badland et al., 2008). In the United States, Frank et al. (2005) and Saelens et al. (2003a) found a positive relationship between neighbourhood walkability and minutes of accelerometer-based moderate physical activity per day. Frank et al. (2005) reported that adults living in neighbourhoods in the highest walkability index quartile were 2.4 times more likely to accumulate 30 min of moderate intensity physical activity per day than those living in neighbourhoods in the lowest walkability index quartile. Saelens et al. (2003a) found a difference of $52 \mathrm{~min} /$ week in moderate intensity physical activity between residents of high and low walkable neighbourhoods. Also in the United States, a strong association between the walkability index and minutes/ 
week of active transportation (walking and cycling), measured by the long version of the International Physical Activity Questionnaire (IPAQ), was reported by Frank et al. (2006). In Australia, Owen et al. (2007) found a positive association between neighbourhood walkability and weekly frequency of walking for transport, also measured by the long version of the IPAQ. No significant associations between walkability and duration of walking for transport were found. In New Zealand, Badland et al. (2008) found that adults commuting through highly connected streets were 6.9 times more likely to engage in transport-related physical activity to get to their workplace than those commuting through poorly connected streets.

Since ecological models suggest that physical activity behaviour depends on a combined action of psychosocial, demographic and physical environmental factors, it is important to investigate not only physical environmental determinants, but also the interactions with psychosocial and demographic factors, in order to develop interventions for behaviour change (Trost et al., 2002). The moderating effect of demographic variables on the relation between physical environmental factors and physical activity in adults has been investigated before (Spence et al., 2006; Parks et al., 2003). However, to our knowledge, no studies have examined interactions between psychosocial correlates and the physical environment in relation to physical activity in adults. But a few studies have investigated the relative influence of psychosocial and physical environmental factors on physical activity behaviour (Giles-Corti and Donovan, 2003; De Bourdeaudhuij et al., 2005). In Australia, Giles-Corti and Donovan (2003) found that in adults the relative influences of psychosocial and physical environmental factors on walking were nearly of equal importance. Achieving recommended levels of walking was associated with high perceived behaviour control, high intention to be physically active, having good access to attractive open spaces and living in an aesthetically pleasing neighbourhood. In Europe (Belgium and Portugal), De Bourdeaudhuij et al. (2005) found that walking/cycling for transportation and walking for recreation in adults were related to social support, walkability and walking facilities in the neighbourhood. Recreational physical activity was mainly determined by social support, self-efficacy and perceived benefits and barriers. The variance in physical activity explained by physical environmental factors was lower (1\%-8\%) than by psychosocial factors (maximum $42 \%$ ).

It is obvious that no definite conclusions can be drawn yet concerning the relative influences of psychosocial and physical environmental factors on walking and other forms of physical activity. Results also differ between countries, so additional research is certainly needed. Most of the studies on walkability and its influence on walking and active transport have been performed in Australia or in the United States and research on this topic still remains sparse. Physical environmental characteristics are very different in Europe, compared to the United States and Australia. For example, in Europe, neighbourhoods with city blocks built in streets with grid patterns are not common. Consequently, additional studies on the relationship between walkability and physical activity are certainly necessary, especially in Europe. Therefore, the present study was executed in Belgium, in the centre of Europe. Since ecological research, including environmental, psychosocial and demographic factors, is still growing and additional studies are needed to improve evidence, this study adds considerably to the literature, focusing on both environmental and psychosocial factors and their impact on physical activity behaviour.

Considering the limitations of the current literature, the main aims of the present study were (1) to investigate whether Belgian adults living in a high walkable neighbourhood are more physically active than those living in a low walkable neighbourhood and (2) to study whether the relationship between the walkability of the neighbourhood and physical activity is different for adults with a high score on transport-related psychosocial correlates, compared to adults with a low score on these correlates.

\section{Methods}

\section{Procedures}

The study was conducted in Sint-Niklaas, Flanders. Flanders is the Dutch-speaking part of Belgium. Sint-Niklaas is a metropolitan city with approximately 70,000 inhabitants and a population density of 832 inhabitants per square kilometre. As a comparison, the population density of Brussels (capital city) is 4441 inhabitants per square kilometre and the population density of Ghent (metropolitan city) is 1468 inhabitants per square kilometre. Data collection took place in November and December 2006. In these months, the mean temperature in Belgium is $5.1^{\circ} \mathrm{C}$, and it rains 13 days per month on average. Two neighbourhoods with a maximal contrast in walkability and a similar number of inhabitants were selected. The walkability index used for neighbourhood selection was based on geographical map data of neighbourhood connectivity and residential density (Frank et al., 2004). Street plans were used to measure connectivity. Residential development maps from the land register were used to determine residential density. Connectivity was defined as 'number of intersections with three or more intersecting streets per square kilometre'. Residential density was assessed as 'number of households per square kilometre' (Leslie et al., 2007). Both the high and the low walkable neighbourhood contained approximately 1500 inhabitants, but due to its lower density, the physical size of the low walkable neighbourhood was larger. A map image of the neighbourhoods is provided in Fig. 1.

In each neighbourhood, one hundred fifty letters with information on the study were randomly posted. Two to six days after posting the information letter, potential participants were visited at home. Up to three attempts were made, on different days and different times of the day, to find someone at home. During the home visit, the researcher asked if someone in the household met the inclusion criteria, including the following: between 20 and 65 years of age, living in private dwellings such as houses and apartments, able to walk without assistance and able to complete questionnaires in Dutch. Adults who met the criteria and agreed to participate were asked to complete a written informed consent form, an adjusted version of the Neighbourhood Physical Activity Questionnaire (NPAQ) (Giles-Corti et al., 2006) and a questionnaire on psychosocial and sociodemographic correlates of physical activity (De Bourdeaudhuij and Sallis, 2002). Participants were asked to fill in the questionnaires independently. All participants were also instructed to wear a pedometer on the right hip for seven consecutive days. They were asked to reset the pedometer to zero at the beginning of each day, to remove the pedometer only while bathing, showering or swimming and to complete an activity log at the end of each day. The completed questionnaires, activity logs and pedometers were collected during a second home visit, one week after the first home visit.

Home visits were done until 60 participants were found in each neighbourhood.

\section{Instruments}

Pedometers. The Yamax Digiwalker SW-200 pedometer (Yamax, Tokyo, Japan) was used in the present study to collect objective 


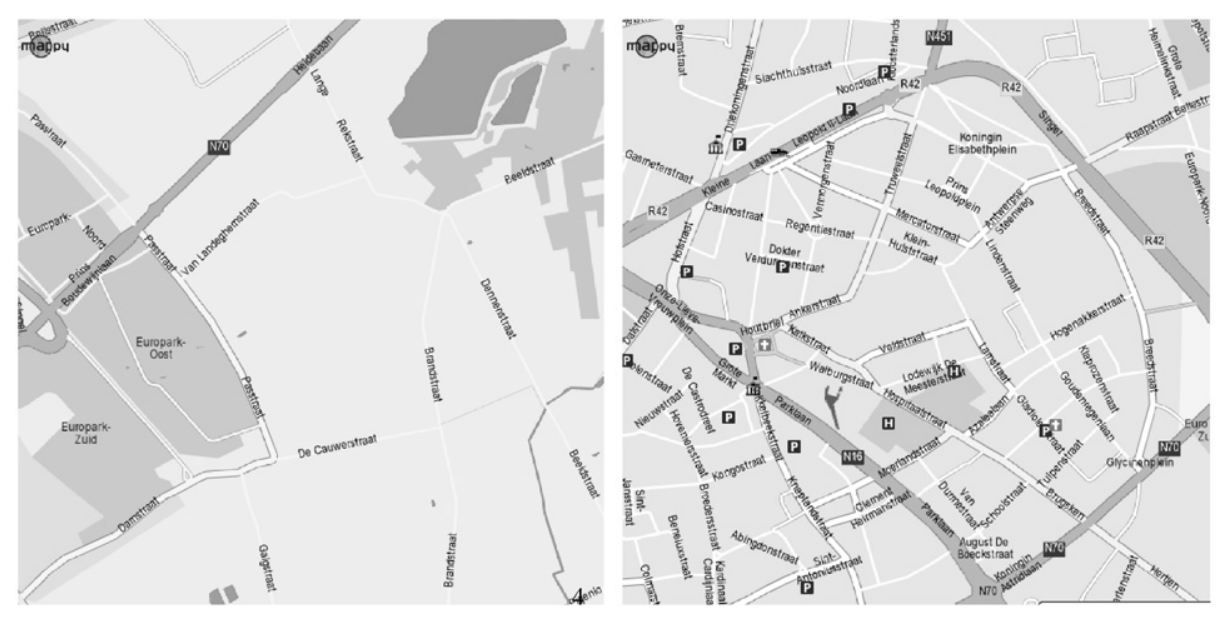

Fig. 1. Map image of the high and low walkable neighbourhood.

data on physical activity. According to the literature, the Yamax pedometer is reliable and accurate for counting steps. Reliability coefficients range between .56 and .99 (Welk et al., 2000; Schneider et al., 2003). Also, the association between step counts and self-reported amounts of physical activity is found to be good (De Cocker et al., 2007).

Activity log. Participants were asked to complete a daily activity $\log$ for the seven consecutive days they wore the pedometer. They were requested to fill in the total number of steps taken each day, as well as to report the time spent cycling and swimming. One hundred fifty steps were imputed at the end-day number of steps for every minute of biking or swimming reported (Miller et al., 2006; De Cocker et al., 2007).

Physical activity questionnaire. Subjective information on walking and cycling for recreation and transport inside and outside the neighbourhood was obtained using a modified version of the NPAQ usual week version. This questionnaire is based on the International Physical Activity Questionnaire (IPAQ) and the Active Australia Survey and was developed for the RESIDential Environment Project (RESIDE) in Australia (Giles-Corti et al., 2006). The questionnaire has acceptable reliability, with kappa coefficients ranging from .67 to .95 (Giles-Corti et al., 2006). 'Neighbourhood' was defined as 'the direct environment, everywhere within a 10-15 min walk of your home'. First, frequency (number of days per week) and duration (hours and minutes per day) of walking for transport in the neighbourhood were queried. Then, these questions were repeated for walking for transport outside the neighbourhood, walking and cycling for recreation in and outside the neighbourhood and cycling for transport in and outside the neighbourhood. In this study, the NPAQ was used instead of the IPAQ because it was necessary to distinguish between activities in and outside the neighbourhood. Physical activity outside the neighbourhood is a common behaviour in Belgium, but is probably not related to the neighbourhood walkability.

Psychosocial variables. The psychosocial questions all targeted cycling and walking for transport as goal behaviour, as previous studies showed that more specific determinant questions are more valid and more able to explain variance (Sallis and Owen, 1999). The questions were adopted from a previous study in adults (De Bourdeaudhuij and Sallis, 2002), and adjusted for this study. Preference for active or passive transport was assessed by asking 'which transport mode do you prefer to go somewhere?' (car/motorbike/public transportation/bike/on foot). Self-efficacy was assessed by the question 'How difficult do you find it to go somewhere by bike or on foot?', social support by 'does your family or do your friends support you travelling by bike or on foot?'. Perceived benefits of physical activity were assessed by the question 'do you think going somewhere by bike or on foot would give you many benefits?'; perceived barriers by asking 'do you experience many barriers to go somewhere on foot or by bike?'; and intention to walk or cycle more when living in a high walkable neighbourhood (intention) by the question 'If you lived in a neighbourhood where it were more convenient to travel on foot or by bike, do you think you would walk or cycle more for transport?'. Response categories for the questions concerning selfefficacy, social support, perceived benefits, perceived barriers and intention where 'not at all/not much/neutral/somewhat/certainly'. The questionnaire only consisted of six questions because previous research showed that these are the key questions regarding psychosocial correlates of physical activity in adults (De Bourdeaudhuij and Sallis, 2002).

Due to lack of normality in the responses for preference for active or passive transport, self-efficacy, social support, perceived benefits, perceived barriers and intention to walk or cycle, dichotomous variables were constructed. The responses "not at all, not much, neutral" were grouped, versus the responses "somewhat and certainly".

Demographic variables. Self-reported demographic variables included sex, age, education, living situation, working situation, working status, height, weight and address.

Observation scale. To obtain objective information on physical environmental factors, an observation scale was used. This observation scale was based on the scale used in the Neighbourhood Quality of Life Study (NQLS) in the United States (www.ipenproject.org) and a scale developed by Brownson et al. (2004). Field observations of aspects concerning street connectivity, land use mix diversity, land use mix access, aesthetics, transport system and traffic safety were executed. 'Land use mix access' was defined as the objective distance to the closest commercial, recreational and educational facilities, 'land use mix diversity' as the objective number of residential, commercial, educational and recreational facilities in a radius of $800 \mathrm{~m}$.

Each participant's neighbourhood was observed. The observation area was defined by an $800 \mathrm{~m}$ radius around a respondent's residence. The $800 \mathrm{~m}$ radius was used to enhance comparability with the questionnaire, where the environment was defined as 'everywhere within a $10-15 \mathrm{~min}$ walk of your home'. This 10-15 min walking distance matches well with an $800 \mathrm{~m}$ radius. All observations were executed by one research team member.

To verify the contrast in walkability between the high and low walkable neighbourhoods prior to the start of the data collection, 
observational data from the two neighbourhoods concerning land use mix access, land use mix diversity, aesthetics, transport system, traffic safety and connectivity, were compared. This was done to increase the accuracy of the walkablity index, because the index primarily used to select the neighbourhoods was only based on paper map data of residential density and connectivity, and these are not the only determinants of neighbourhood walkability.

All observational data on the physical environment significantly differed between the two neighbourhoods. Table 1 summarizes differences (independent sample t-tests) in land use mix access, land use mix diversity, street connectivity, aesthetics, transport system and traffic safety.

In the high walkable neighbourhood, shorter distances from one's residence to commercial, educational and recreational facilities, more stores (bakeries, butchers, supermarkets, banks and clothes shops), more residences (houses and apartments), more schools and a greater number of sport and non-sport facilities were observed than in the low walkable neighbourhood. There were also more intersections and culs-de-sac in the high walkable neighbourhood, more green spaces (with possibilities for walking and cycling), more trees and benches along the streets, more bus stops, more streets with a bike-way, streets with a sidewalk and streets with a combination of bike-way and sidewalk, more crosswalks, more traffic lights and there was also a train station.

\section{Data analysis}

All analyses were conducted using SPSS 12.0 for Windows. To analyse differences in step counts, walking and biking for transport/recreation in and outside the neighbourhood between the two neighbourhoods, independent sample $t$-tests were used. Two-way ANOVAs were executed to investigate the interactions between the transport-related psychosocial correlates and the physical environment in relation to physical activity. Mean step counts per day and minutes per week of walking/cycling for transport in the neighbourhood were successively entered as dependant variables. Neighbourhood was a fixed factor in every analysis and the dichotomised psychosocial variables were successively added as fixed factors. As prior chi-square tests and independent sample $t$-tests showed that the samples of the high and low walkable neighbourhood were comparable on all demographic characteristics, no covariates were included in the analyses. Statistical significance was set at a level of .05 .

\section{Results}

\section{Demographic characteristics of the sample}

In total, 120 20-65 year old adults participated in the present study. The overall response rate was $47.8 \%$ (120/251). The response rate was $52.6 \%(60 / 114)$ in the high walkable neighbourhood and $43.8 \%(60 / 137)$ in the low walkable neighbourhood. Main reasons for not participating in the study were: not interested (62.6\%); not at home (31.3\%) and not meeting the inclusion criteria (6.1\%). The sample consisted of 55 men (age: $\mathrm{M}=42.8$ years, $\mathrm{SD}=13.9 ; \mathrm{BMI}: \mathrm{M}=25.3 \mathrm{~kg} / \mathrm{m}^{2}, \mathrm{SD}=2.5$ ) and 65 women (age: $\mathrm{M}=43.1$ years, $\mathrm{SD}=12.7$; $\mathrm{BMI}: \mathrm{M}=23.7 \mathrm{~kg} / \mathrm{m}^{2}$, $\mathrm{SD}=3.5)$. The mean age of the total sample was $43.0(\mathrm{SD}=13.3)$

Table 1

Observational data of two neighbourhoods

\begin{tabular}{|c|c|c|c|}
\hline & $\begin{array}{l}\text { Low walkable neighbourhood } \\
\text { Mean (SD) }\end{array}$ & $\begin{array}{l}\text { High walkable neighbourhood } \\
\text { Mean (SD) }\end{array}$ & $t(p)$ \\
\hline \multicolumn{4}{|c|}{ Land use mix access (objective distance to facilities) } \\
\hline \multicolumn{4}{|c|}{$1 \geqslant 2500 \mathrm{~m} ; 2 \geqslant 1500 \mathrm{~m},<2500 \mathrm{~m} ; 3 \geqslant 700 \mathrm{~m},<1500 \mathrm{~m} ; 4 \geqslant 200 \mathrm{~m},<700 \mathrm{~m} ; 5=\leqslant 200 \mathrm{~m}$} \\
\hline Commercial & $1.93(.59)$ & $4.68(.25)$ & $32.86^{* * *}$ \\
\hline Recreational (sport) & $1.77(.30)$ & $3.65(.82)$ & $16.74^{* * *}$ \\
\hline Recreational (non sport) & $2.12(.58)$ & $3.69(.78)$ & $12.46^{* * *}$ \\
\hline Educational & $1.72(.39)$ & $4.64(.39)$ & $41.39 * * *$ \\
\hline \multicolumn{4}{|c|}{ Land use mix diversity (number in a radius of $800 \mathrm{~m}$ ) } \\
\hline Houses & $416.18(110.83)$ & $1574.00(119.18)$ & $55.11^{* * *}$ \\
\hline Apartments & $5.23(1.23)$ & $66.63(5.02)$ & $92.00 * * *$ \\
\hline Stores & $2.12(1.54)$ & $56.15(29.74)$ & $14.05^{* * *}$ \\
\hline Schools & $.63(.84)$ & $7.25(3.04)$ & $16.25^{* * *}$ \\
\hline Recreational facilities & $1.67(1.13)$ & $11.57(2.94)$ & $24.33^{* * *}$ \\
\hline \multicolumn{4}{|l|}{ Street connectivity (number in a radius of $800 \mathrm{~m}$ ) } \\
\hline Intersections & $11.90(3.07)$ & $39.12(7.28)$ & $26.67^{* * *}$ \\
\hline Cul-de-sacs & $3.62(1.32)$ & $4.40(1.95)$ & $2.58^{*}$ \\
\hline \multicolumn{4}{|l|}{ Aesthetics } \\
\hline Green spaces (number in a radius of $800 \mathrm{~m}$ ) & $1.00(.00)$ & $2.50(.75)$ & $15.54 * * *$ \\
\hline Trees & $.70(.46)$ & $1.58(.50)$ & $10.08^{* * *}$ \\
\hline$(0=$ no, $1=$ few, $2=$ many $)$ & & & \\
\hline Benches $(0=$ no, $1=$ few, $2=$ many $)$ & $.00(.00)$ & $1.58(.50)$ & $24.67^{* * *}$ \\
\hline \multicolumn{4}{|l|}{ Transport system (number in a radius of $800 \mathrm{~m}$ ) } \\
\hline Bus stops & $6.78(1.47)$ & $27.73(13.06)$ & $12.35^{* * *}$ \\
\hline Train station & $.00(.00)$ & $.52(.50)$ & $7.94^{* * *}$ \\
\hline Streets with bike-way & $3.63(1.03)$ & $16.08(1.74)$ & $47.76^{* * *}$ \\
\hline Streets with sidewalk & $3.23(.59)$ & $20.25(2.36)$ & $54.28^{* * *}$ \\
\hline Streets with bike-way and sidewalk & $3.23(.59)$ & $16.75(4.12)$ & $25.18^{* * *}$ \\
\hline \multicolumn{4}{|l|}{ Traffic safety (number in a radius of $800 \mathrm{~m}$ ) } \\
\hline Crosswalks & $6.50(3.01)$ & $39.93(14.15)$ & $17.90^{* * *}$ \\
\hline Traffic lights & $1.10(.60)$ & $9.53(3.33)$ & $19.32^{* * *}$ \\
\hline
\end{tabular}


years. The mean body mass index (BMI) was $24.5 \mathrm{~kg} / \mathrm{m}^{2}$ $(\mathrm{SD}=3.2)$. There were no significant gender differences in age or BMI. Of the total sample, $9.2 \%$ obtained a primary school degree only, $44.5 \%$ had a secondary school degree and $46.2 \%$ a college or university degree. Of all participants, $72.5 \%$ was employed; $71.9 \%$ reported working as a white-collar worker, $28.1 \%$ as a blue-collar worker. Mean step counts of the total sample was 8707 $(\mathrm{SD}=3098)$ steps/day. All participants reported pedometer data for at least four days. Based on the recommendations of TudorLocke and colleagues (2005), who concluded that a minimum of three days of pedometer data is sufficient to estimate adult pedometer-determined physical activity in a week, all participants were included in the dataset.

Analyses on gender, age, BMI, years of education, working status and working situation showed no significant differences between participants of the high walkable and the low walkable neighbourhood. Demographic information of the participants of both neighbourhoods can be found in Table 2 .

\section{Differences in physical activity between the neighbourhoods (Table 3)}

Participants living in the high walkable neighbourhood took significantly more steps per day $(M=9318$ steps/day, $S D=3055)$ than those living in the low walkable neighbourhood $(M=8096$

Table 2

Descriptive characteristics for the high and low walkable neighbourhood

\begin{tabular}{lll}
\hline & $\begin{array}{l}\text { High walkable } \\
\text { neighbourhood }\end{array}$ & $\begin{array}{l}\text { Low walkable } \\
\text { neighbourhood }\end{array}$ \\
\hline Sex (\%) & & \\
$\quad$ Female & 56.7 & 51.7 \\
Male & 43.3 & 48.3 \\
Age (mean (SD)) & $42.3(12.9)$ & $45.7(13.5)$ \\
Education (\%) & & \\
$\quad$ Primary & 6.7 & 11.9 \\
Secondary & 51.6 & 37.4 \\
College/university & 41.7 & 50.7 \\
Employment status (\%) & & 71.7 \\
$\quad$ Employed & 73.3 & 28.3 \\
$\quad$ Not employed & 26.7 & \\
Occupation (\%) & & 33.3 \\
$\quad$ Blue collar & 22.7 & 66.7 \\
$\quad$ White collar & 77.3 & $23.6(4.2)$ \\
Body mass index (mean (SD)) & & $25.1(2.0)$ \\
$\quad$ Females & $23.9(2.8)$ & \\
Males & $25.5(3.0)$ & \\
\hline
\end{tabular}

steps/day, $\mathrm{SD}=3044)(t=2.19, p=.03)$. For the adjusted NPAQ a significant difference between the two neighbourhoods was found for walking for transport inside the neighbourhood. Adults living in the high walkable neighbourhood reported more walking for transport in the neighbourhood $(M=104.33 \mathrm{~min} /$ week, $S D=$ 95.10) than those living in the low walkable neighbourhood $(\mathrm{M}=22.83 \mathrm{~min} / \mathrm{week}, \mathrm{SD}=61.00)(t=5.59, p<.001)$. For walking for transport outside the neighbourhood, cycling for transport in and outside the neighbourhood and walking and cycling for recreation in and outside the neighbourhood, no significant differences were found between the two neighbourhoods.

Because of the skewness of the physical activity data, non parametric analyses were also executed (Mann-Whitney $U$ test). The results of these tests were similar to the results described above. Interactions between neighbourhood walkability and transport-
related psychosocial correlates in relation to physical activity

As the psychosocial questionnaire focused specifically on factors related to active transport in the neighbourhood, the step counts and walking and cycling for transport in the neighbourhood were used as outcome variables for this research question.

For mean steps per day, a significant interaction was found between the walkability of the neighbourhood and preference for active/passive transport ( $F=5.97, p=.02$ ) (Fig. 1$)$. In adults with a preference for passive transport, mean step counts were dependant on the walkability of the neighbourhood. More specifically, adults with a preference for passive transport took significantly more steps per day when living in the high walkable neighbourhood $(M=9385$ steps/day, $S D=2962)$ than when living in the low walkable neighbourhood $(M=7197$ steps/day, $S D=2567)$ $(t=3.51, p<.001)$. In adults with a preference for active transport, no significant differences in step counts between the high walkable neighbourhood ( $M=9184$ steps/day, $S D=3308)$ and the low walkable neighbourhood ( $\mathrm{M}=9767$ steps/day, $\mathrm{SD}=3211)$ were found $(t=.57)$.

A significant interaction was also found between the walkability of the neighbourhood and the intention to walk or cycle more when living in a high walkable neighbourhood $(F=5.80$, $p=.02$ ) (Fig. 2). For adults with a low intention to walk or cycle, mean step counts were dependant on the walkability of the neighbourhood. Thus, adults with a low intention to walk or cycle took significantly more steps per day when living in the high walkable neighbourhood $(\mathrm{M}=9844, \mathrm{SD}=3094)$ than when living in the low walkable neighbourhood $(M=7582, S D=2771)$ $(t=3.12, p=.003)$. For adults with a high intention to walk or cycle, no significant differences in step counts between the high

Table 3

Differences in physical activity between the neighbourhoods

\begin{tabular}{|c|c|c|c|}
\hline & $\begin{array}{l}\text { Low walkable neighbourhood } \\
\text { Mean (SD) }\end{array}$ & $\begin{array}{l}\text { High walkable neighbourhood } \\
\text { Mean (SD) }\end{array}$ & $t(p)$ \\
\hline Steps/day & $8096(3044)$ & $9318(3055)$ & $2.19^{*}$ \\
\hline Walking transport in neighbourhood (min/week) & $22.83(61.00)$ & $104.33(95.10)$ & $5.59 * * *$ \\
\hline Walking transport outside neighbourhood (min/week) & $40.00(122.99)$ & $55.00(135.42)$ & .64 \\
\hline Cycling transport in neighbourhood (min/week) & $92.42(178.78)$ & 74.08 (113.69) & .67 \\
\hline Cycling transport outside neighbourhood (min/week) & $89.42(172.96)$ & $51.50(107.42)$ & 1.44 \\
\hline Walking recreation in neighbourhood (min/week) & $36.08(86.08)$ & $46.00(121.51)$ & .52 \\
\hline Walking recreation outside neighbourhood (min/week) & $70.00(111.58)$ & $58.18(106.12)$ & .59 \\
\hline Cycling recreation in neighbourhood ( $\mathrm{min} /$ week) & $42.92(113.03)$ & $27.00(79.94)$ & .89 \\
\hline Cycling recreation outside neighbourhood (min/week) & $58.08(133.26)$ & $23.17(48.17)$ & 1.91 \\
\hline
\end{tabular}

$\mathrm{ns}=$ Not significant $(p<.05) .^{* *} p \leqslant .01$.

$* p \leqslant .05$.

$* * * \quad p \leqslant .001$. 


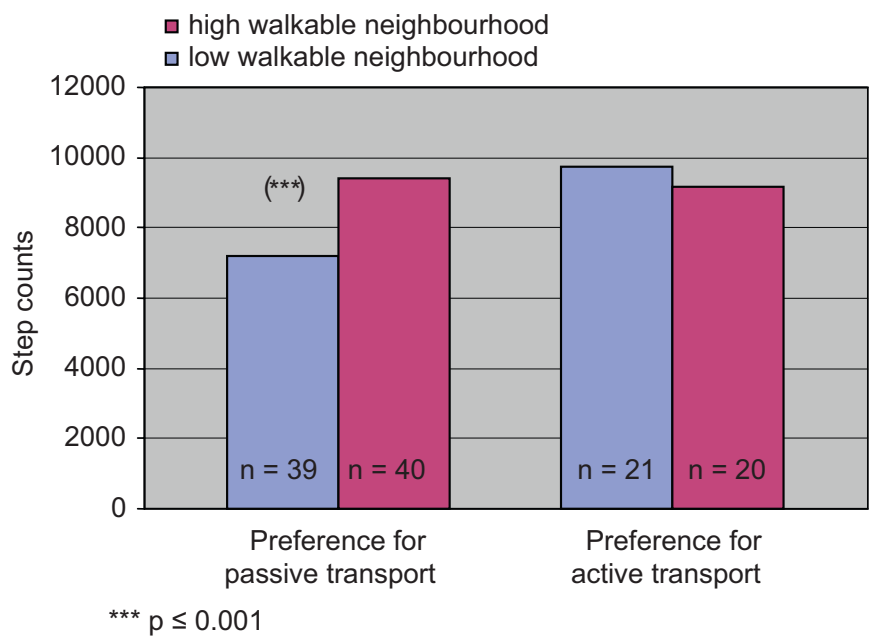

Fig. 2. Interactions between preference for active or passive transport and neighbourhood walkability for mean step counts.

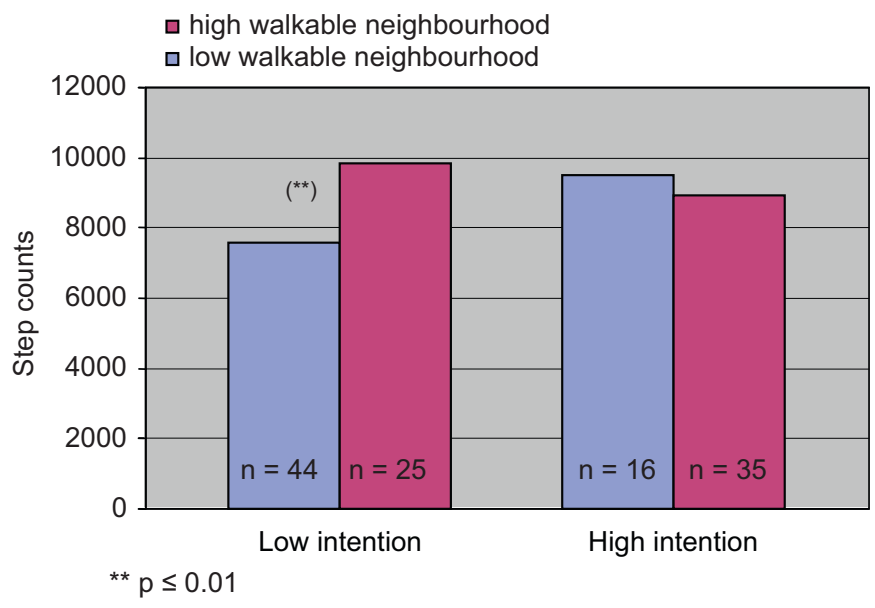

Fig. 3. Interactions between intention to walk or cycle and neighbourhood walkability for mean step counts.

walkable neighbourhood $(\mathrm{M}=8942, \mathrm{SD}=3015)$ and the low walkable neighbourhood $(M=9511, S D=3395)$ were found $(t=.60)$ (Fig. 3).

For the interactions between the walkability of the neighbourhood and self-efficacy, social support, perceived benefits and perceived barriers in relation to mean step counts per day, no significant results were found. Furthermore, no significant interactions were found for reported minutes of walking and cycling for transport in the neighbourhood.

\section{Discussion}

The first aim of the present study was to investigate whether adults living in a high walkable neighbourhood were more physically active than adults living in a low walkable neighbourhood. Results from Australia (Owen et al., 2004; Owen et al., 2007) and the United States (Saelens et al., 2003b; Frank et al., 2005, 2006) were supported by this Belgian study. The main finding of this study was that neighbourhood walkability, based on objective attributes concerning street connectivity and residential density and observed land use mix, was associated with residents' step counts and walking for transport in the neighbourhood. This confirms previous studies in the transport literature, revealing that residents in denser communities with greater street connectivity and more land use mix reported higher rates of walking/cycling for utilitarian purposes than residents from lowdensity, poorly connected and single land use neighbourhoods (Saelens et al., 2003b; Frank et al., 2006). In the present study, both reported minutes of walking for transport and number of step counts differed between residents of the high and low walkable neighbourhood. Inhabitants of the high walkable neighbourhood took on average 9300 steps/day and reported about 100 min of walking for transport per week, while residents of the low walkable neighbourhood took an average 8100 steps per day and reported only $23 \mathrm{~min}$ of walking for transport per week. As no significant differences in cycling for transport, and walking and cycling for recreation were found, the differences in step counts between adults living in the high walkable neighbourhood and those living in the low walkable neighbourhood could be mainly a reflection of the difference in walking for transport.

The neighbourhood observations revealed several physical environmental factors that could also explain the differences in step counts and walking for transport between the two neighbourhoods. First, there were significantly more streets with a sidewalk in the high walkable neighbourhood, so the availability of pedestrian infrastructure was better in that neighbourhood. This can encourage the inhabitants to walk more for transport. Previous studies (Giles-Corti and Donovan, 2003; Owen et al., 2004; Wendel-Vos et al., 2007) found positive associations between the availability of pedestrian infrastructure and walking for transport. Secondly, a greater availability of commercial, educational and recreational facilities and shorter distances to these facilities were observed in the high walkable neighbourhood. The presence of these facilities could have a positive impact on walking for transport, as inhabitants can reach these nearby facilities on foot. Also, the greater availability of recreation facilities in the high walkable neighbourhood could enhance sports participation next to active transport. However, the NPAQ only focuses on walking and cycling for transport and no information on sports participation was collected in this study.

No differences in cycling for transport were found between the two neighbourhoods, although differences in cycling infrastructure between the high and low walkable neighbourhood were observed. There are several possible explanations for this finding. First, it could be due to the lack of specificity of the walkability index. Even though the cycling infrastructure was included, it is known that the walkability index concentrates more on walking attributes than on cycling attributes (Boer et al., 2007; Rodriguez et al., 2006). Secondly, adults usually cycle for transport when covering rather large distances. This means that the radius used in this study for the definition of the neighbourhood and for the observation of walkability, may be too narrow and therefore not relevant for investigating cycling behaviour. To improve the opportunities to study the relation between walkability or bikability of the neighbourhood and cycling behaviour in adults, the radius of neighbourhoods should be enlarged to approximately $4 \mathrm{~km}$ around a residence ( $15 \mathrm{~min}$ of cycling). Third, since cycling is strongly supported in Belgium, it is possible that other factors are more related to cycling behaviour than physical environmental characteristics, such as the financial incentives offered to employees for cycling to work or the generally positive attitude towards cycling among Flemish adults.

The entire study sample took on average 8707 (3098) steps/day and $36.7 \%$ of the sample reached the 10,000 steps/day standard on an average day. When comparing these results to other pedometer-based studies, the sample was more active than 
populations in the United States (Tudor-Locke et al., 2004; Wyatt et al., 2005), but less active than adults who participated in a large scale Belgian study, where the mean daily step count was 9650 (4520) (De Cocker et al., 2007). This could possibly be due to the timing of data collection in this study. Data were collected during winter months, when physical activity levels are consistently lower than during summer and spring (Matthews et al., 2001). In the large scale Belgian pedometer study, data were collected during springtime. Nonetheless, these findings suggest that the physical activity levels of the present study sample were representative. It had been expected that participants in this study would mainly be people interested in physical activity, who are more active than the average Flemish adult. The results show that this was not the case.

The second aim of this study was to investigate whether the relation between the walkability of the neighbourhood and physical activity was different for adults with a low score on transport-related psychosocial correlates, compared to adults with a high score on these correlates. Results showed that for adults with a preference for passive transport and/or a low intention to walk or cycle, living in a high walkable neighbourhood was associated with taking more steps. Persons with a preference for passive transport took on average 2000 more steps per day when living in a high walkable neighbourhood than when living in a low walkable neighbourhood. Similar results were found regarding intention to walk or cycle. Adults with low intentions and living in a high walkable neighbourhood took about 2000 more steps per day than those with similar intentions who were living in a low walkable neighbourhood. This is very hopeful, as several intervention studies have shown that an increment of 2000-2500 steps/day can improve important health outcomes, for example lower body weight and lower cholesterol levels (Hills et al., 2003; http:// aom.americaonthemove.org). For participants with a preference for active transport and/or with a high intention to walk or cycle, number of step counts did not differ significantly between the two neighbourhoods, but was generally high. Also, in the low walkable neighbourhood, adults with a high intention and a preference for active transport took significantly more steps than those with a low intention and a preference for passive transport. This is a logical finding, because in low walkable neighbourhoods, the physical environment is not appealing and does not facilitate physical activity. Therefore, in these environments, mainly psychosocial determinants such as attitudes are important and adults with positive attitudes towards active transport will take more steps than those with less positive attitudes.

The main finding from these results is that neighbourhood walkability appears to be especially important to people with a less positive attitude towards active transport and/or a lower intention to walk or cycle, promoting them to use active transport. This is a major finding and establishes the importance of supportive environments in a health promotion context. Because the present study had a cross-sectional nature and this is the first study showing these effects, no definite conclusions on this topic can be drawn yet. Still, the results are promising when thinking in terms of health promotion and physical activity interventions. Adults with less positive scores on psychosocial correlates are an important and difficult target group for physical activity promotion who can be reached by creating an activity enhancing environment. For this group, interventions changing the physical environment could be very effective as an additional intervention mode, along with individual-based interventions. In that way, health promotion could take place simultaneously on different levels and the effects on physical activity behaviour could be greater. In the same line, Giles-Corti (2006) stated that interventions targeting both people and places are required to increase physical activity levels. She found that the likelihood of walking at recommended levels was nearly eight times higher in adults with both a supportive environment and positive psychological cognitions compared with those low on both. Many other researchers emphasise the importance of broader, multilevel, ecological approaches to physical activity promotion (Spence and Lee, 2003; Sallis and Owen, 1999).

It is difficult to compare these findings with results of other studies, because only one study investigated comparable transport-related psychosocial factors (Frank et al., 2007). That study, conducted in the United States, showed a different trend than the findings of the present study. Frank and colleagues (2007) investigated the interactions between neighbourhood preference and walkability on physical activity. They found that for adults who did not prefer to live in a high walkable neighbourhood, the amount of walking, measured through a 2-day recall diary, was extremely low regardless of neighbourhood walkability. Only $3 \%$ of the participants living in a low walkable neighbourhood and 7\% of those living in a high walkable neighbourhood reported at least one walking trip. For adults who preferred to live in a high walkable neighbourhood, more participants reported walking trips when living in a high walkable neighbourhood (33.9\%) than when living in a low walkable neighbourhood (16.0\%). Consequently, the authors suggested that providing more walkable environments could result in increased walking, but only for adults with a high preference to live in a high walkable neighbourhood. The differences in the results between the present study and the study of Frank and colleagues (2007) could be due to measurement issues or cultural differences between the United States and Belgium. Another possible explanation is the relativity of the 'walkability' concept. In Belgium, neighbourhood walkability is generally rather high. Therefore, high walkable neighbourhoods in the United States will probably be low walkable according to Belgian standards. This may limit the comparability between results of Belgian studies and studies executed in the United States. Consequently, it can be suggested that more research in different countries and more standardised protocols for investigating interactions between psychosocial correlates and walkability are certainly needed to make it possible to draw definite conclusions.

The present study has some limitations. A first limitation is the cross-sectional nature of the study, which means that causal relations can not be determined. At this stage of research on physical environmental determinants of physical activity, crosssectional studies are needed to determine the most promising environmental correlates and their interactions with other variables. Once these correlates are determined, they can be evaluated in prospective studies (Sallis and Owen, 2002). A second limitation is the fact that the study was conducted in one city and that only two neighbourhoods were included. This limits the opportunity to generalise the results for Belgium. Third, concerning the measurement tools, no GIS was used in this study, as in Europe GIS is often not available yet. Instead, field observations were executed to obtain an objective rating of the physical environment. For the observations, a radius buffer was applied. In future research, when GIS is more common in Belgium, a network buffer should be used, because it is a more accurate method to asses distance. Fourth, the use of pedometers has some limitations. Pedometers can not collect data on swimming, cycling and static activities. In this study, we tried to solve this limitation by using an activity log additional to the pedometer, but this log can not solve all the pedometer's limitations. Fifth, no information on participants' car availability and ownership was collected. Car availability has consistently been shown to be related to travel behaviour (Badland and Schofield, 2008) and can differ by neighbourhood walkability, so this could be a confounder of the relationship between walkability and active transport. Sixth, as 
$71.9 \%$ of the participants reported working as a white-collar worker, the study sample was highly educated. This can influence physical activity levels, as many studies showed that highly educated persons usually are more physically active (Trost et al., 2002). However, as physical activity levels (step counts) of participants in this study were lower than those found in other studies in Flanders (De Cocker et al., 2007), representativity of the physical activity levels was supported despite the highly educated nature of the sample.

The strengths of this study firstly included the use of both objective (pedometer) and subjective (questionnaire) measures of physical activity. By using pedometers, objective data on physical activity (steps) were collected and by using the physical activity questionnaire, more specific information on the context of physical activity could be evaluated. Secondly, individual and neighbourhood level SES did not differ between the two neighbourhoods. Both the high and the low walkable neighbourhood were relatively high SES areas. Therefore, SES could not confound the relationship between the walkability and physical activity behaviour.

For future research, it is important that more neighbourhoods with a wide variety in walkability are investigated. Also, the use of geographic information systems (GIS) should be encouraged to evaluate the objective physical environment. Most studies in the United States and Australia use GIS to determine walkability, but in Europe, and certainly in Belgium, the use of GIS in health research is not common yet. For example, De Bourdeaudhuij and colleagues $(2003,2005)$ already studied the relationship between physical environmental characteristics and physical activity in Belgium, and also used the neighbourhood environmental walkability scale (NEWS) questionnaire and observational data instead of GIS to evaluate physical environmental characteristics. Nevertheless, some digital map data are available for most cities in Belgium, so GIS applications show potential. It would also be very interesting, especially in Belgium and in other countries where cycling is promoted, to investigate the 'bikability' of neighbourhoods and to relate this concept with cycling behaviour in adults. To do this, the radius of selected neighbourhoods needs to be larger and specific objective environmental parameters concerning cycling infrastructure should be defined.

To conclude, we would like to emphasise the importance of research regarding physical environmental factors once more. If future longitudinal studies can confirm causal relations between environmental characteristics and physical activity, interventions for health promotion can be developed. The main advantage of interventions changing the physical environment is that the entire population living in the target neighbourhoods can be influenced by the intervention. As a consequence, even small effects on physical activity are very beneficial, because many people are affected (Owen et al., 2004). Moreover, physical environmental changes are expected to be relatively permanent, so long term effects on physical activity can be expected. Finally, walkability and other physical environmental factors appear to be mainly associated with walking for transport. Walking is also the most common form of physical activity in adults (Rafferty et al., 2002). Consequently, physical environmental interventions are of particular relevance as walking and active transport are among the most promising behaviours for increasing physical activity in adults. In this context, future interventions have the potential to reduce automobile dependency and to create a healthier, more ecological and safer environment.

\section{References}

Badland, H., Schofield, G.M., 2008. Understanding the relationships between private automobile availability, overall physical activity, and travel behavior in adults. Transportation 35, 363-374.
Boer, R., Zheng, Y., Overton, A., Ridgeway, G.K., Cohen, D.A., 2007. Neighbourhood design and walking trips in ten US metropolitan areas. American Journal of Preventive Medicine 32, 298-304.

Brownson, R.C., Hoehner, C.M., Brennan, L.K., Cook, R.A., Elliott, M.B., McMullen, K., 2004. Reliability of two instruments for auditing the environment for physical activity. Journal of Physical Activity \& Health 1,191-208.

De Bourdeaudhuij, I., Sallis, J., 2002. Relative contribution of psychosocial variables to the explanation of physical activity in three population-based adult samples. Preventive Medicine 34, 279-288.

De Bourdeaudhuij, I., Teixeira, P.J., Cardon, G., Deforche, B., 2005. Environmental and psychosocial correlates of physical activity in Portuguese and Belgian adults. Public Health Nutrition 8, 886-895.

De Cocker, K., Cardon, G., De Bourdeaudhuij, I., 2007. Pedometer-determined physical activity an its comparison with the International Physical Activity Questionnaire in a sample of Belgian adults. Research Quarterly for Exercise and Sport 78, 429-437.

Dishman, R.K., Sallis, J.F., 1994. Determinants and interventions for physical activity and exercise. In: Bouchard, C., Shephard, R.J., Stephens, T. (Eds.), Physical Activity, Fitness and Health: International Proceedings and Consensus Statement. Human Kinetics, Champaign, IL, pp. 214-238.

European Commission, 2003. Eurobarometer Physical Activity, Special Eurobarometer 183-6, Wave 58.2, European Opinion Research Group EEIG, Directorate General Health and Consumer Protection and Directorate General Press and Communication, pp. 1-49.

Forsyth, A., D'Sousa, E., Koepp, J., Oakes, J.M., Schmitz, K.H., Van Riper, D., Zimmerman, J., 2007. Twin cities walking study: Environment and physical activity: GIS protocols, Version 4.1. (work in progress) 〈www.designcenter.umn.edu >.

Frank, L.D., Andresen, M.A., Schmid, T.L., 2004. Obesity relationships with community design, physical activity and time spent in cars. American Journal of Preventive Medicine 27, 87-96.

Frank, L.D., Schmid, T.L., Sallis, J.F., Chapman, J., Saelens, B.E., 2005. Linking objectively measured physical activity with objectively measured urban form: findings from SMARTRAQ. American Journal of Preventive Medicine 28 (2S2), $117-125$.

Frank, L.D., Sallis, J.F., Conway, T.L., Chapman, J.E., Saelens, B.E., Bachman, W., 2006. Many pathways from land use to health: associations between neighborhood walkability and active transportation, body mass index and air quality. Journal of the American Planning Association 72, 75-87.

Frank, L.D., Saelens, B.E., Powell, K.E., Chapman, J.E., 2007. Stepping towards causation: do built environments or neighbourhood and travel preferences explain physical activity, driving and obesity? Social Science and Medicine 65 , 1898-1914.

Giles-Corti, B., 2006. People or places, what should be the target? Journal of Science and Medicine in Sport 9, 357-366.

Giles-Corti, B., Donovan, R.J., 2003. Relative influences of individual, social environmental and physical environmental correlates of walking. American Journal of Public Health 93, 1583-1589.

Giles-Corti, B., Timperio, A., Cutt, H., Pikora, T., Bull, F., Knuiman, M., Bulsara, M., Van Niel, K., Shilton, T., 2006. Development of a reliable measure of walking within and outside the local neighbourhood: RESIDE's Neighborhood Physical Activity Questionnaire. Preventive Medicine 42, 455-459.

Haskell, W.L., Min Lee, I., Pate, R.R., Powell, K.E., Blair, S.N., Franklin, B.A., Macera, C.A., Heath, G.W., Thompson, P.D., Bauman, A., 2007. Physical activity and public health: updated recommendation for adults from the American College of Sports Medicine and the American Heart Association. Medicine \& Science in Sports \& Exercise 39 (8), 1423-1434.

Hills, J.O., Wyatt, H.R., Reed, G.W., Peters, J.C., 2003. Obesity and the environment: where do we go from here? Science 299, 853-855.

King, A.C., Jeffery, R.W., Fridinger, F., Dusenbury, L., Provence, L., Hedlund, S.A., Spangler, K., 1995. Physical activity: issues and opportunities. Health Education Quarterly 22 (4), 499-511.

Leslie, E., Coffee, N., Frank, L., Owen, N., Bauman, A., Hugo, G., 2007. Walkability of local communities: using Geographic Information Systems to objectively assess relevant environmental attributes. Health \& Place 13, 111-122.

Matthews, C.E., Freedson, P.S., Hebert, J.R., Stanek, E.J., Merriam, P.A., Rosal, M.C. Ebbeling, C.B., Ockene, I.S., 2001. Seasonal variation in household, occupational and leisure time physical activity: longitudinal analyses from the seasonal variation of blood cholesterol study. American Journal of Epidemiology 153, 172-183.

Miller, R., Brown, W., Tudor-Locke, C., 2006. But what about swimming and cycling? how to count non-ambulatory activity when using pedometers to assess physical activity? Journal of Physical Activity and Health 3, 257-266.

Owen, N., Humpel, N., Leslie, E., Bauman, A., Sallis, J., 2004. Understanding environmental influences on walking: review and research agenda. American Journal of Preventive Medicine 27, 67-76.

Owen, N., Cerin, E., Leslie, E., duToit, L., Coffee, N., Frank, L.D., Bauman, A.E., Hugo, G., Saelens, B.E., Sallis, J.F., 2007. Neighborhood walkability and the walking behavior of Australian adults. American Journal of Preventive Medicine 32, 387-395.

Parks, S.E., Housemann, R.A., Brownson, R.C., 2003. Differential correlates of physical activity in urban and rural adults of various socioeconomic backgrounds in the United States. Journal of Epidemiology and Community Health $57,29-35$

Rafferty, A.P., Reeves, M.J., McGhee, H.B., 2002. Physical activity patterns among walkers and compliance with public health recommendations. Medicine and Science in Sports and Exercise 34, 1255-1261. 
Rodriguez, D.A., Khattak, A.J., Evenson, K.R., 2006. Can new urbanism encourage physical activity? Journal of the American Planning Association 72, 43-54.

Saelens, B.E., Sallis, J.F., Black, J.B., Chen, D., 2003a. Neighborhood-based differences in physical activity: an environment scale evaluation. Research and Practice 93, 1552-1558.

Saelens, B.E., Sallis, J.F., Frank, L.D., 2003b. Environmental correlates of walking and cycling: findings from transportation, urban design and planning literatures. Annals of Behavioral Medicine 25, 80-91.

Sallis, J., Owen, N., 1999. Physical Activity and Behavioral Medicine. Sage Publications, Thousand Oaks, CA.

Sallis, J., Owen, N., 2002. Ecological models of health behaviour. In: Glanz, K., Rimer, B., Lewis, M. (Eds.), Health Behaviour and Health Education: Theory, Research and Practice. Jossey-Bass, San Francisco, pp. 462-484.

Schneider, P.L., Crouter, S.E., Lukajic, O., Bassett, D.R., 2003. Accuracy and reliability of 10 pedometers for measuring steps over a 400-m walk. Medicine and Science in Sports and Exercise 35, 1779-1784.

Spence, J.C., Lee, R.E., 2003. Toward a comprehensive model of physical activity. Psychology of Sport and Exercise 4, 7-24.

Spence, J.C., Plotnikoff, R.C., Rovniak, L.S., Ginis, K.A.M., Rodgers, W., Lear, S.A., 2006. Perceived neighbourhood correlates of walking among participants visiting the Canada on the Move website. Canadian Journal of Public Health-Revue Canadienne de Santé Publique 97 (S1), S36-S40.
Trost, S.G., Owen, N., Bauman, A.E., Sallis, J.F., Brown, W., 2002. Correlates of adults participation in physical activity: review and update. Medicine \& Science in Sports \& Exercise 34, 1996-2001.

Tudor-Locke, C., Ham, S.A., Macera, C.A., Ainsworth, B.E., Kirtland, K.A., Reis, J.P., et al., 2004. Descriptive epidemiology of pedometer-determined physical activity. Medicine \& Science in Sports \& Exercise 36, 1567-1573.

Tudor-Locke, C., Burkett, L., Reis, J.P., Ainsworth, B.E., Macera, C.A., Wilson, D.K. 2005. How many days of pedometer monitoring predict weekly physical activity in adults? Preventive Medicine 40, 293-298.

US Department of Health and Human Services, 1996. Physical activity and health: a report of the Surgeon General. Atlanta, GA: US Department of Health and Human Services, Centers for Disease Control and Prevention, National Center for Chronic Disease Prevention and Health Promotion.

Welk, G.J., Differding, J.A., Thompson, R.W., Blair, S.N., Dziura, J., Hart, P., 2000. The utility of the Digi-Walker step counter to assess daily physical activity patterns. Medicine \& Science in Sports \& Exercise 32 (9), S481-S488.

Wendel-Vos, W., Droomers, M., Kremers, S., Brug, J., Van Lenthe, F., 2007. Potential environmental determinants of physical activity in adults: a systematic review. Obesity Reviews 8, 425-440.

Wyatt, H.R., Peters, J.C., Reed, G.W., Barry, M., Hill, J.O., 2005. A Colorado statewide survey of walking and its relation to excessive weight. Medicine \& Science in Sports \& Exercise 37, 724-730. 